\title{
Hippocampal High-Frequency Stimulation Inhibites the Progression of Rapid Kindling-Induced Seizure in Rats*
}

\author{
Belen Gori ${ }^{1}$, Magdalena Pereyra ${ }^{1}$, Lucas Toibaro ${ }^{1}$, Carola Brescacin ${ }^{1}$, Gerardo Battaglia ${ }^{2}$, \\ Julieta Pastorino $^{1}$, Ariela Smigliani ${ }^{1}$, Milagros Galardi ${ }^{1}$, Silvia Kochen ${ }^{1 \#}$ \\ ${ }^{1}$ Center of Clinical and Experimental Neuroscience, Epilepsy, Cognition and Behavior, Institute of Cellular Biology and Neurosci- \\ ence "Prof. E. De Robertis" (IBCN)-National Council for Scientific and Technical Research (CONICET), School of Medicine, Uni- \\ versity of Buenos Aires, Buenos Aires, Argentina; ${ }^{2}$ Laboratory of Biomedical Technology, Department of Electronics, Buenos Aires \\ Faculty, National Technological University (UTN), Buenos Aires, Argentina. \\ Email: "\#skochen@retina.ar
}

Received March 22 $2^{\text {nd }}, 2013$; revised April 26 $6^{\text {th }}, 2013$; accepted May $16^{\text {th }}, 2013$

Copyright (C) 2013 Belen Gori et al. This is an open access article distributed under the Creative Commons Attribution License, which permits unrestricted use, distribution, and reproduction in any medium, provided the original work is properly cited.

\begin{abstract}
Epilepsy is one of the most common serious neurological disorders. Pharmacoresistant epilepsy patients are poorly controlled or their seizures are refractory to drug treatment. Resective surgery is frequently a promising therapy in this population, however, not all the patients meet the eligibility criteria for the surgical treatment. Deep brain stimulation has been investigated in clinical studies and animal studies as an alternative treatment, but the optimal stimulation parameters remain an issue. The present study was designed to investigate the effect of unilateral high-frequency stimulation (HFS) of hippocampus on seizure development by using the hippocampal rapid kindling method (hRK) in rats, and compared the results with those of low-frequency stimulation previously published by our group. We used male Wistar rats implanted with electrodes in the ventral hippocampus. All rats underwent hRK (biphasic square wave pulses, $20 \mathrm{~Hz}$ for 10 seconds) during three consecutive days (twelve stimulations per day). The control group ( $h R K ; n=7$ ) received only RK stimulus, while the treated group (HFS-hRK; $n=9$ ) received also HFS (biphasic square wave pulses, $130 \mathrm{~Hz}$ for 30 seconds) immediately before the RK stimulus, during three consecutive days. At the end of behavioral testing $78 \%(\mathrm{p}<0.01)$ of the animals receiving HFS treatment were still not fully kindled staying in stages $0-$ III $(\mathrm{p}<0.01)$. HFS group needed a higher number of stimulations to achieve stage III $(p<0.05)$ with respect to control group. However, no significant differences in the cumulative daily afterdischarge duration were observed. HFS did not present significant differences compared with LFS in any of studied parameters. The findings suggest that unilateral HFS applied on hippocampus effectively inhibited the epileptogenic process induced by hippocampal rapid kindling. According to the comparative results about hippocampal rapid kindled animals stimulated with HFS and LFS (5 Hz), we found no conclusive information on which treatment is most efficient.
\end{abstract}

Keywords: Hippocampal Rapid Kindling; Epilepsy; Electrical Stimulation; High-Frequency Stimulation; Low-Frequency Stimulation

\section{Introduction}

Epilepsy is one of the most common serious neurological disorders with a prevalence of $1 \%-2 \%$ of general population [1]. Pharmacoresistant epilepsy in adults, since one third of the patients are poorly controlled or their seizures are refractory to drug treatment [2]. Resective surgery is frequently a promising therapy in this population, however, it has been estimated that over $40 \%$ of patients do not meet the eligibility criteria for the surgical treatment

\footnotetext{
*This study was funded by UBACYT, School of Medicine, University of Buenos Aires.

\#Corresponding author.
}

[2]. Since the early 1970s, deep brain stimulation (DBS) has been investigated in clinical studies and animal studies as an alternative treatment. The stimulation of hippocampus may provide an alternative to patients who have refractory TLE [3-11]. Despite this broad range of researches, the optimal stimulation parameters as well as the neuroanatomical target remain an issue.

Kindling is one of the most widely used models for TLE [12-14]. Traditional kindling could have some limitations, such as the long time required (10 - 30 days depending of anatomical target) for the rats to become fully kindled and the fact that rapid growth of brain and skull 
of animals inevitably displaces the electrodes [15]. During the last two decades, rapid kindling (RK) has been developed as an alternative to traditional kindling. In the RK model the animal is brought from the naive to the epileptic state within a matter of several hours [16$18]$.

Several studies using traditional kindling in different anatomical targets have evaluated the inhibitory effect of low frequency stimulation [19-23] and high-frequency stimulation [24-26]. Few reports have been carried out in hippocampus using RK model $[25,27,28]$, moreover for its role in epileptogenesis [29-31].

This study was tested the efficacy of unilateral highfrequency stimulation (HFS) on hippocampus using the RK model and compared with our previous results on low frequency stimulation (LFS) in the same model [28].

\section{Materials and Methods}

\subsection{Animals}

Male Wistar rats (220 - $270 \mathrm{~g}$ ) were treated according to guidelines approved by the European Ethics Commitee (86/609/EEC) and the ethical guidelines of the Committee of Health Guide for the Care and Use of Laboratory Animals of School of Veterinary Medicine of Buenos Aires University. The experimental protocol was approved by the Institutional Committee for the Care and Use of Laboratory Animals (CICUAL) of School of Medicine of Buenos Aires University. Furthermore, efforts were made to minimize the number of animals used in the study and their suffering. The animals were housed individually in cages and kept under environmentally controlled conditions $\left(23^{\circ} \mathrm{C}-25^{\circ} \mathrm{C}, 12 \mathrm{~h}\right.$ normal light/ dark cycles (lights on from 6:00-18:00 h)). Animals were provided with water and food ad libitum. They were acclimated for 7 - 10 days before surgery. Experiments were carried out each day between 10:00-17:00 $\mathrm{h}$.

\subsection{Surgery}

The surgery was carried out according to Toibaro et al. [28]. The rats were deeply anaesthetized with intraperitoneal injections of a ketamine/xylazine mixture at a concentration of $85.0 \mathrm{mg} / \mathrm{ml}$ ketamine and $15.0 \mathrm{mg} / \mathrm{ml}$ xylazine. The rats were fixed in a stereotaxic frame according to the method of Paxinos and Watson 1998 [32] and the skull was exposed. Five holes were drilled, two of them for anchor screws, two for placement of a stainless steel wire used as ground and reference and one for placement of the electrode. A bipolar recording and stimulating electrode $(0.2 \mathrm{~mm}$ in diameter) was chronically implanted in the right ventral hippocampus, using the following coordinates in $\mathrm{mm}$ from bregma and skull surface: anteroposterior -5.3 ; lateral 5.2; depth 7.5 [32].
The electrodes consisted in two twisted stainless steel Teflon-coated wires (tip distance $0.5-1.0 \mathrm{~mm}$ ) insulated except for $0.5 \mathrm{~mm}$ at the tip. Electrode was connected to a miniature receptacle, which was embedded in the skull with dental cement. At least 7 - 10 days were allowed for recovery from surgery before starting the experiment. Following completion of the experimental protocol, all animals were anaesthetized by an intraperitoneal injection of ketamine $(85 \mathrm{mg} / \mathrm{ml})$ and xylazine $(15 \mathrm{mg} / \mathrm{ml})$ and then transcardially perfused with $4 \%$ paraformaldehyde. Brains were frozen and coronally sectioned into $40 \mu \mathrm{m}$ slices with a microtome and slide mounted, allowing for confirmation of electrode placements. The data of the animals with false placement of their electrode position or existence of any abnormality, such as lesion, were not included in the results.

\subsection{Stimulation Procedures}

All the recordings were performed after the rat had been transferred from the home cage to a recording box $(30 \times$ $30 \times 30 \mathrm{~cm}$ ). The head-stage of the rat was connected to a flexible, shielded cable. Evoked responses were recorded in the freely moving rat while the animal was awake with its eyes open.

On day 0 , seven to ten days post surgical recovery, the afterdischarge threshold (ADT) for each animal was determined (Figure 1). The ADT is the minimum intensity that evokes an afterdischarge (AD) of $5 \mathrm{~s}$ or longer, defined as a two-fold increase in the EEG baseline. An initial current of $50 \mu \mathrm{A}$ (peak-to-base; $2 \mathrm{~s}$ trains of biphasic square wave pulses, $20 \mathrm{~Hz}$ ) was applied and then increased in steps of $50 \mu \mathrm{A}$ (to a maximum of $400 \mu \mathrm{A}$ ), with 5 minutes intervals between current delivery. The animals that did not elicit $\mathrm{AD}$ with a current intensity of

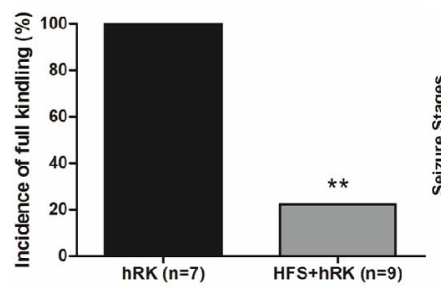

(a)

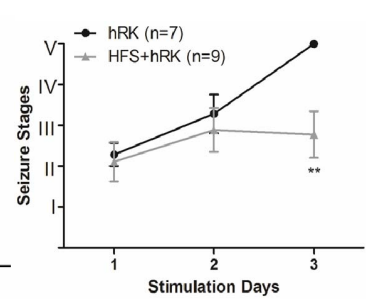

(b)
Figure 1. Effect of HFS on seizure severity. (a) Effect of HFS on the incidence of full kindling (FK) state in hippocampal-kindled rats. Chi-square test showed that HFStreated group (HFS + hRK; light gray bar) had significantly less incidence of FK than control group (hRK; black bar; ${ }^{* *}$ p $<0.01$ ), reaching FK only $22.2 \%$ of HFS + hRK animals; (b) Effect of HFS on progression of behavioral seizure stages during 3 days of stimulations in HFS + hRK compared to control group (hRK). The nonparametric MannWhitney $U$ test showed a significant difference on behavioral seizure scores on day $3\left(^{* *} \mathrm{p}<0.01\right)$. Data were shown as mean \pm SEM. 
$400 \mu \mathrm{A}$ were excluded from the experiment.

On day 1 , the animals were assigned to two groups: the control group ( $h R K ; n=7)$ and the group treated with HFS (HFS + hRK; $n=9$ ). All animals received the rapid kindling stimulus. The hRK stimulus consisted in 12 daily stimulations during three consecutive days. Each stimulus was carried out at ADT intensity with $10 \mathrm{~s}$ trains of biphasic square wave pulses, at a frequency of $20 \mathrm{~Hz}$ with intervals of 30 minutes between each of them. The HFS (biphasic square wave pulses $50 \mu \mathrm{A}, 130 \mathrm{~Hz}$ for 30 seconds) was applied to the treated group immediately before each one of the 12 RK stimulations on three stimulation days. HFS parameters were determined according to Goodman et al. 2005 [19] and our preliminary experiments [28].

Electroencephalogram (EEG) during each $\mathrm{AD}$ was recorded through the hippocampal electrodes. All of the recording were obtained and reviewed by using a digital system (Stellate-Bioscience system, Bioscience S.R.L, Buenos Aires, Argentina). The signal was amplified 1000 times, filtered $0.5-40 \mathrm{~Hz}(3 \mathrm{~dB} /$ octave), and digitized at a sampling rate of $200 \mathrm{~Hz}$. The behavioral manifestation was classified following an adjusted version of the scale of Racine [33: Racine et al., 1972]: stage I: immobility, facial clonus, wet dog shakes; stage II: head nodding, chewing, automa-tisms; stage III: clonus of one forelimb; stage IV: rearing, bilateral forelimb clonus; stage V: rearing, bilateral fore-limb clonus, loss of balance and falling [34-36]. Stages I - III were considered as focal seizures, while stages IV and $\mathrm{V}$ were considered as generalized seizures (GS) [22]. When the animals exhibited three consecutive stage 5 seizures, they were regarded as fully kindled. An investigator who had no information about the animal group and the number of stimulations scored the behavioral responses. After the completion of the experiment, the incidence of full kindling, the mean of the number of stimulations necessaries to reach the stage IV and $\mathrm{V}$ (generalization of seizures), the behavioral progression of kindling (stages I $\mathrm{V}$; according to Racine's scores), and the cumulative daily $\mathrm{AD}$ duration (ADD) were monitored to evaluate the effect of the HFS during the kindling acquisition. Values for ADD were calculated by adding the duration of ADs recorded after each of the daily twelve stimulations.

\subsection{Statistical Analysis}

A two-way analysis of variance (ANOVA) followed by a post hoc Bonferroni's test was done to compare the ADD and the number of stimulations required to achieve different stages between hRK (control) and HFS+hRK groups. The behavioral progression of kindling (stages I V) was analyzed by using Mann Whitney U test. In the case of comparing generalized seizure incidence, chisquare test was used. Data are expressed as mean and standard error of the mean (SEM). Statistical analysis was carried out by GraphPad Prism 4.0 for Windows. Statistical comparisons between HFS and LFS data were performed using one way ANOVA followed by Student-Newman-Keuls post test. For all analysis, the tests were performed two-sided and a $\mathrm{p}<0.05$ was considered significant.

\section{Results}

\subsection{Effect of High-Frequency Stimulation}

All the animals used in this experiment fulfilled the above mentioned criteria, 7 rats were assigned to the control group (hRK) and 9 rats to the treated group (HFS $+\mathrm{hRK})$. The following parameters were analyzed: incidence of full kindling, seizure stage achieved per day, number of stimulations needed to reach the different stages and daily afterdischarge duration (ADD).

At the end of the experimental protocol, all control rats were fully kindled, while in HFS group only 2 of 9 rats $(22.2 \%)$ became fully kindled $(\mathrm{p}<0.01$; Chi-square test; Figure 1(A)). The seizure stage achieved by the HFS + hRK group was significantly lower $(\mathrm{p}<0.01$; Mann Whitney $U$ test) than the control group (hRK) on the third day, as shown in Figure 1(B).

HFS group required a higher number of stimulations to reach stage III $(12.4 \pm 2.6 ; \mathrm{p}<0.05$; two-way ANOVA followed by post hoc Bonferroni's test) compared to control animals $(5.0 \pm 1.6)$. This effect was also observed to achieve stage IV-V, but without reaching significance (HFS: $17.4 \pm 2.9$; hRK: $13.7 \pm 1.9 ; \mathrm{p}>0.05$ ) (Figure 2).

Regarding ADD, HFS group showed a non significant decreased duration from second and third day in relation to control group.

\subsection{Comparative Analysis between High- and Low-Frequency Stimulation on Seizure Parameters}

The HFS group showed a lower incidence of full kin-

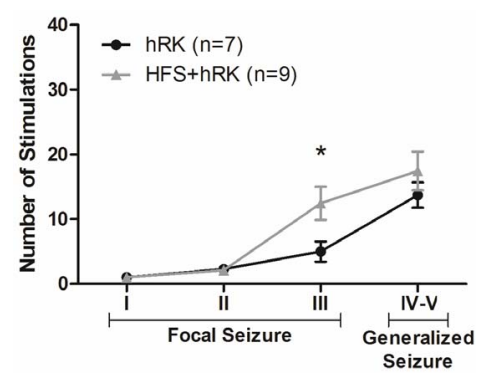

Figure 2. Effect of HFS on number of stimulations needed to achieve each seizure stage. A two-way ANOVA followed by a post hoc Bonferroni's test showed that the average number of stimulations required to reach stage III was significantly increased in HFS + hRK group $(n=9)$ compared with control group (hRK; $\left.n=7 ;{ }^{*} \mathbf{p}<0.05\right)$. Data were shown as mean \pm SEM. 
dling when compared to LFS group, but did not reach significance (Figure 3).

We did not observe any differences in relation to the daily achieved seizure stage, between HFS and LFS treatments (LFS data previously published by our group [28]; Figure 1(b) for HFS).

ADD has not been significantly modified by HFS neither LFS. Despite both HFS and LFS show a decreased ADD.

The animals of HFS group $(n=9)$ have achieved stage IV - V after $17.4 \pm 2.9$ stimulations, while animals of LFS group $(\mathrm{n}=7)$ have needed more stimulations $(26.1 \pm$ $3.9)$ to reach the same seizure stages $(p<0.01)$ (LFS data previously published by our group [28]; Figure 2 for HFS).

\section{Discussion}

The results obtained show that HFS significantly reduced the incidence of full kindling. In relation to behavioral test, on the third day $78 \%$ of animals treated with HFS remained on stages 0 - III, equivalent to focal seizures. HFS could interfere with the course of epileptogenesis, delaying the progression of behavioral seizure stage and avoiding generalized seizures. This decrease in the behavioral response is in agreement with results reported by Cuellar-Herrera et al. [24], in which the same frequency was used, and by Zhang et al. [26] despite the fact that a higher frequency $(200 \mathrm{~Hz})$ was delivered.

We observed an evident, but not significant, decreased ADD in the group treated with HFS, other reports found similar results, but HFS effect was weaker than our findings $[25,26]$. In this study, we used HFS parameters closely related to those employed in epilepsy patients [33,37-39] and to the ones considered safe in the treatment of Parkinson's disease [40,41]. The values are also in agreement with those used in experimental epilepsy by Wyckhuys et al. [25,27].

One of the objectives of this study was to compare the

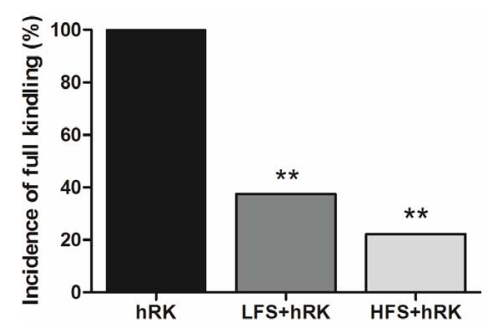

Figure 3. Comparison of HFS and LFS effect on the incidence of full kindling. A one-way ANOVA followed by a Newman-Keuls post test showed that both LFS and HFS treatment significantly decreased $\left({ }^{* *} \mathrm{p}<0.01\right)$ the incidence of full kindling compared to control group. The statistical analysis did not show significance between HFS and LFS treatments, but a lower incidence of full kindling in HFS group was observed. ${ }^{1}$ LFS data previously published by our group [28]. effect of LFS, previously published by our group [28], with the data of HFS obtained in this report. The results of both, HFS and LFS pre-treatment, did not present significant differences between them in any of studied parameters. HFS group seems to be more efficient than LFS regarding to the incidence of full kindling. HFS group required higher number of stimulations to achieving stage III, however in LFS group was necessary a higher number of stimulations to achieve behavioral stages IV - V than HFS. Both treatment groups seemed to interfere on the progression of seizure stages and did not significantly affect the ADD, even though HFS showed an inhibition of increase of the ADD. Further studies are needed to conclude which treatment is most effective.

An in vitro study which compared the effects of prolonged LFS and HFS on epileptiform activity, suggested that both LFS and HFS were effective in suppressing epileptogenic progress, but mediated through different mechanisms [42]. A few studies using LFS in the kindling focus have indicated that LFS-induced long-term depression or depotentiation may reverse kindling-induced long-term potentiation in the focus, so retarding seizure development [43-45]. On the other hand, HFS $(130 \mathrm{~Hz})$ can evoke long-term Potentiation [45-47] which in turn can change the functional organization of the hippocampal network [48]. However, these mechanisms are not mutually exclusive and may even operate at different times. These different mechanisms could underlie the differences in effectiveness between HFS and LFS observed in this report.

Finally, we conclude that unilateral HFS $(130 \mathrm{~Hz})$ applied on hippocampus effectively inhibited the epileptogenic process induced by hippocampal rapid kindling. According to the comparative results about hippocampal rapid kindled animals stimulated with HFS $(130 \mathrm{~Hz})$ and LFS $(5 \mathrm{~Hz})$, we found no conclusive results on which treatment is most efficient.

\section{REFERENCES}

[1] T. Dua, H. M. de Boer, L. L. Prilipko and S. Saxena, "Epilepsy Care in the World: Results of an ILAE/IBE/ WHO Global Campaign against Epilepsy Survey," Epilepsia, Vol. 47, No. 7, 2006, pp. 1225-1231. doi:10.1111/j.1528-1167.2006.00595.x

[2] P. Kwan, A. Arzimanoglou, A. T. Berg, M. J. Brodie, W. A. Hauser, G. Mathern, S. L. Moshé, E. Perucca, S. Wiebe and J. French, "Definition of Drug Resistant Epilepsy: Consensus Proposal by the Ad Hoc Task Force of the ILAE Commission on Therapeutic Strategies," Epilepsia, Vol. 51, No. 6, 2010, pp. 1069-1077. doi:10.1111/j.1528-1167.2009.02397.x

[3] I. S. Cooper, I. Amin, M. Riklan, J. M. Waltz and T. P. Poon, "Chronic Cerebellar Stimulation in Epilepsy. Clinical and Anatomical Studies," Archives of Neurology, 
Vol. 33, No. 8, 1976, pp. 559-570. doi:10.1001/archneur.1976.00500080037006

[4] A. R. Upton, I. S. Cooper, M. Springman and I. Amin, "Suppression of Seizures and Psychosis of Limbic System Origin by Chronic Stimulation of Anterior Nucleus of the Thalamus," International Journal of Neurology, Vol. 19-20, 1985-1986, pp. 223-230.

[5] F. Velasco, M. Velasco, C. Ogarrio and G. Fanghanel, "Electrical Stimulation of the Centromedian Thalamic Nucleus in the Treatment of Convulsive Seizures: A Preliminary Report," Epilepsia, Vol. 28, No. 4, 1987, pp. 421-430. doi:10.1111/j.1528-1157.1987.tb03668.x

[6] M. Velasco, F, Velasco, A. L. Velasco, F. Jiménez, F. Brito and I. Márquez, "Acute and Chronic Electrical Stimulation of the Centromedian Thalamic Nucleus: Modulation of Reticulo-Cortical Systems and Predictor Factors for Generalized Seizure Control," Archives of Medical Research, Vol. 31, No. 3, 2000, pp. 304-315. doi:10.1016/S0188-4409(00)00085-0

[7] M. Velasco, F. Velasco and A. L. Velasco, "Centromedian-Thalamic and Hippocampal Electrical Stimulation for the Control of Intractable Epileptic Seizures," Journal of Clinical Neurophysiology, Vol. 18, No. 6, 2001, pp. 495-513. doi:10.1097/00004691-200111000-00001

[8] A. Schulze-Bonhage, "Deep Brain Stimulation: A New Approach to the Treatment of Epileps," Deutsches Ärzteblatt International, Vol. 106, No. 24, 2009, pp. 407-412.

[9] B. C. Jobst, "Brain Stimulation for Surgical Epilepsy," Epilepsy Research, Vol. 89, No. 1, 2010, pp. 154-161. doi:10.1016/j.eplepsyres.2009.08.017

[10] SANTE Study Group, "Electrical Stimulation of the Anterior Nucleus of Thalamus for Treatment of Refractory Epilepsy," Epilepsia, Vol. 51, No. 5, 2010, pp. 899-908. doi:10.1111/j.1528-1167.2010.02536.x

[11] T. Tykocki, T. Mandat, A. Kornakiewicz, H. Koziara and P. Nauman, "Deep Brain Stimulation for Refractory Epilepsy," Archives of Medical Science, Vol. 8, No. 5, 2012, pp. 805-816. doi:10.5114/aoms.2012.31135

[12] G. V. Goddard, D. C. McIntyre and C. K. Leech, "A Permanent Change in Brain Function Resulting from Daily Electrical Stimulation," Experimental Neurology, Vol. 25, No. 3, 1969, pp. 295-330. doi:10.1016/0014-4886(69)90128-9

[13] G. V. Goddard, "The Kindling Model of Epilepsy," Trends in Neurosciences, Vol. 6, 1983, pp. 275-279. doi:10.1016/0166-2236(83)90118-2

[14] J. O. McNamara, D. W. Bonhaus and C. Shin, "The Kindling Model of Epilepsy," In: P. A. Schwartzkroin, Ed., Epilepsy: Models, Mechanisms, and Concepts, Cambridge University Press, Cambridge, 1993, pp. 27-47. doi:10.1017/CBO9780511663314.003

[15] A. Mazarati, D. Shin, S. Auvin, R. Caplan and R. Sankar, "Kindling Epileptogenesis in Immature Rats Leads to Persistant Depressive Bahavior," Epilepsy and Behavior, Vol. 10, No. 3, 2007, pp. 377-383. doi:10.1016/j.yebeh.2007.02.001

[16] E. W. Lothman, J. M. Hatlelid, C. F. Zorumski, J. A. Conry, P. F. Moon and J. B. Perlin, "Kindling with Rap- idly Recurring Hippocampal Seizures," Brain Research, Vol. 360, No. 1-2, 1985, pp. 83-91. doi:10.1016/0006-8993(85)91223-5

[17] E. W. Lothman and J. M. Williamson, "Rapid Kindling with Recurrent Hippocampal Seizures," Epilepsy Research, Vol. 14, No. 3, 1993, pp. 209-220. doi:10.1016/0920-1211(93)90045-9

[18] E. W. Lothman and J. M. Williamson, "Closely Spaced Recurrent Hippocampal Seizures Elicit Two Types of Heightened Epileptogenesis: A Rapidly Developing, Transient Kindling and a Slowly Developing, Enduring Kindling," Brain Research, Vol. 649, No. 1-2, 1994, pp. 7184. doi:10.1016/0006-8993(94)91050-2

[19] J. H. Goodman, R. E. Berger and T. K Tcheng, "Preemptive Low-Frequency Stimulation Decreases the Incidence of Amygdala-Kindled Seizures," Epilepsia, Vol. 46, No. 1, 2005, pp. 1-7. doi:10.1111/j.0013-9580.2005.03804.x

[20] L. X. Yang, C. L. Jin, Z. B. Zhu-Ge, S. Wang, E. Q. Wei, I. C. Bruce and Z. Chen, "Unilateral Low-Frequency Stimulation of Central Piriform Cortex Delays Seizure Development Induced by Amygdaloid Kindling in Rats," Neuroscience, Vol. 138, No. 4, 2006, pp. 1089-1096. doi:10.1016/j.neuroscience.2005.12.006

[21] M. Mohammad-Zadeh, J. Mirnajafi-Zadeh, Y. Fathollahi, M. Javan, P. Ghorbani, M. Sadegh and S. M. Noorbakhsh, "Effect of Low Frequency Stimulation of Perforant Path on Kindling Rate and Synaptic Transmission in the Dentate Gyrus during Kindling Acquisition in Rats," Epilepsy Research, Vol. 75, No. 2-3, 2007, pp. 154-161. doi:10.1016/j.eplepsyres.2007.05.003

[22] S. H. Zhang, H. L. Sun, Q. Fang, K. Zhong, D. C. Wu, S. Wang and Z. Chen, "Low-Frequency Stimulation of the Hippocampal CA3 Subfield Is Anti-Epileptogenic and Anti-Ictogenic in Rat Amygdaloid Kindling Model of Epilepsy," Neuroscience Letters, Vol. 455, No. 1, 2009, pp. 51-55. doi:10.1016/j.neulet.2009.03.041

[23] M. Sadegh, J. Mirnajafi-Zadeh and V. Sheibani, "Serine/ Threonine Protein Phosphatases Have No Role in the Inhibitory Effects of Low-Frequency Stimulation in Perforant Path Kindling Acquisition in Rats," Neuroscience Letters, Vol. 451, No. 3, 2009, pp. 266-269. doi:10.1016/j.neulet.2009.01.001

[24] M. Cuellar-Herrera, L. Neri-Bazan and L. L. Rocha, "Behavioral Effects of High Frequency Electrical Stimulation of the Hippocampus on Electrical Kindling in Rats," Epilepsy Research, Vol. 72, No. 1, 2006, pp. 10-17. doi:10.1016/j.eplepsyres.2006.07.002

[25] T. Wyckhuys, T. De Smedt, P. Claeys, R. Raedt, L. Waterschoot, K. Vonck, C. Van den Broecke, C. Mabilde, L. Leybaert, W. Wadman and P. Boon, "High Frequency Deep Brain Stimulation in the Hippocampus Modifies Seizure Characteristics in Kindled Rats," Epilepsia, Vol. 48, No. 8, 2007, pp. 1543-50. doi:10.1111/j.1528-1167.2007.01038.x

[26] Q. Zhang, Z. C. Wu, J. T. Yu, N. N. Yu, X. L. Zhong and L. Tan, "Mode-Dependent Effect of High-Frequency Electrical Stimulation of the Anterior Thalamic Nucleus on Amygdala-Kindled Seizures in Rats," Neuroscience, Vol. 217, 2012, pp. 113-122. 
doi:10.1016/j.neuroscience.2012.05.009

[27] T. Wyckhuys, R. Raedt, K. Vonck, W. Wadman and P. Boon, "Comparison of Hippocampal DBS with High (130 $\mathrm{Hz})$ and Low $(5 \mathrm{~Hz})$ Frequency on Afterdischarges in Kindled Rats," Epilepsy Research, Vol. 88, No. 2-3, 2010, pp. 239-246. doi:10.1016/j.eplepsyres.2009.11.014

[28] L. Toibaro, M. Pereyra, J. Pastorino, A. Smigliani, F. Ocariz, G. Ortmann, M. M. Galardi, M. B. Gori and S. Kochen, "Effect of Unilateral Low-Frequency Stimulation of Hippocampus on Rapid Kindling-Induced Seizure Development in Rats," Neuroscience and Medicine, Vol. 3, No. 2, 2012, pp. 174-180. doi:10.4236/nm.2012.32022

[29] D. King, S. S. Spencer, G. McCarthy, M. Luby and D. D. Spencer, "Bilateral Hippocampal Atrophy in Medial Temporal-Lobe Epilepsy,” Epilepsia, Vol. 36, No. 9, 1995, pp. 905-910. doi:10.1111/j.1528-1157.1995.tb01634.x

[30] T. H. Swanson, "The Pathophysiology of Human Mesial Temporal Lobe Epilepsy," Journal of Clinical Neurophysiology, Vol. 12, No. 1, 1995, pp. 2-22. doi:10.1097/00004691-199501000-00001

[31] S. S. Spencer, "Neural Networks in Human Epilepsy: Evidence of and Implications for Treatment," Epilepsia, Vol. 43, No. 3, 2002, pp. 219-227. doi:10.1046/j.1528-1157.2002.26901.x

[32] G. Paxinos and C. Watson, "The Rat Brain in Stereotaxic Coordinates," 4th Edition, Academic Press, San Diego, 1998.

[33] K. Vonck, P. Boon, E. Achten, J. De Reuck and J. Caemaert, "Long-Term Amygdalohippocampal Stimulation for refractory Temporal Lobe Epilepsy," Annals of Neurology, Vol. 52, No. 5, 2002, pp. 556-565. doi:10.1002/ana.10323

[34] R. J. Racine, "Modification of Seizure Activity by Electrical Stimulation: I. Afterdischarge Threshold and II. Motor Seizure," Electroencephalography and Clinical Neurophysiology, Vol. 32, No. 3, 1972, pp. 269-294. doi:10.1016/0013-4694(72)90176-9

[35] D. C. McIntyre, M. O. Poulter and K. Gilby, "Kindling: Some Old and Some New," Epilepsy Research, Vol. 50, No. 1-2, 2002, pp. 79-92. doi:10.1016/S0920-1211(02)00071-2

[36] M. Sato, R. J. Racine and D. C. McIntyre, "Kindling: Basic Mechanisms and Clinic Validity," Electroencephalography and Clinical Neurophysiology, Vol. 76, No. 5, 1990, pp. 459-472. doi:10.1016/0013-4694(90)90099-6

[37] F. Velasco, M. Velasco, F. Jiménez, A. L. Velasco, F. Brito, M. Rise and J. D. Carrillo-Ruiz, "Predictors in the Treatment of Difficult-to-Control Seizures by Electrical Stimulation of the Centromedian Thalamic Nucleus," Neurosurgery, Vol. 47, No. 2, 2000, pp. 295-305. doi:10.1097/00006123-200008000-00007
[38] A. L. Velasco, M. Velasco, F. Velasco, D. Menes, L. Rocha, M. Briones and I. Márquez, "Subacute and Chronic Electrical Stimulation of the Hippocampus on Intractable Temporal Lobe Seizures: Preliminary Report," Archives of Medical Research, Vol. 31, No. 3, 2000, pp. 316-328. doi:10.1016/S0188-4409(00)00064-3

[39] K. Vonck, P. Boon, P. Claeys, S. Dedeurwaerdere, R. Achten and D. Van Roost, "Long-Term Deep Brain Stimulation for Refractory Temporal Lobe Epilepsy," Epilepsia, Vol. 46, Suppl. 5, 2005, pp. 98-99. doi:10.1111/j.1528-1167.2005.01016.x

[40] L. Vercueil, A. Benazzouz, C. Deransart, K. Bressand, C. Marescaux, A. Depaulis and A. L. Benabid, "High-Frequency Stimulation of the Subthalamic Nucleus Suppresses Absence Seizures in the Rat: Comparison with Neurotoxic Lesions," Epilepsy Research, Vol. 31, No. 1, 1998, pp. 39-46. doi:10.1016/S0920-1211(98)00011-4

[41] A. L. Benabid, "Deep Brain Stimulation for Parkinson's Disease," Current Opinion in Neurobiology, Vol. 13, No. 6, 2003, pp. 696-706. doi:10.1016/j.conb.2003.11.001

[42] B. C. Albensi, G. Ata, E. Schmidt, J. D. Waterman and D. Janigro, "Activation of Long-Term Synaptic Plasticity Causes Suppression of Epileptiform Activity in Rat Hippocampal Slices," Brain Research, Vol. 998, No. 1, 2004, pp. 56-64. doi:10.1016/j.brainres.2003.11.010

[43] L. Velísek, J. Velísková and P. K. Stanton, "Low-Frequency Stimulation of the Kindling Focus Delays Basolateralamygdala Kindling Inimmature Rats," Neuroscience Letters, Vol. 326, No. 1, 2002, pp. 61-63. doi:10.1016/S0304-3940(02)00294-X

[44] S. R. Weiss, A. Eidsath, X. L. Li, T. Heynen and R. M. Post, "Quenching Revisited: Low Level Direct Current Inhibits Amygdala-Kindled Seizures," Experimental Neurology, Vol. 154, No. 1, 1998, pp. 185-192. doi:10.1006/exnr.1998.6932

[45] M. Schubert, H. Siegmund, H. C. Pape and D. Albrecht, "Kindling-Induced Changes in Plasticity of the Rat Amygdala and Hippocampus," Learning and Memory, Vol. 12, No. 5, 2005, pp. 520-526. doi:10.1101/lm.4205

[46] T. V. Bliss and G. L. Collingridge, "A Synaptic Model of Memory: Long-Term Potentiation in the Hippocampus," Nature, Vol. 361, No. 6407, 1993, pp. 31-39. doi:10.1038/361031a0

[47] R. C. Malenka and R. A. Nicoll, "Long-Term Potentiation: A Decade of Progress?" Science, Vol. 285, No. 5435, 1999, pp. 1870-1874. doi:10.1126/science.285.5435.1870

[48] C. J. Behrens, L. P. van den Boom, L. de Hoz, A. Friedman and U. Heinemann, "Induction of Sharp Wave-Ripple Complexes in Vitro and Reorganization of Hippocampal Networks," Nature Neuroscience, Vol. 8, No. 11, 2005, pp. 1560-1567. doi:10.1038/nn1571 